\title{
World Congress on Osteoporosis, Osteoarthritis and Musculoskeletal Diseases (WCO-IOF-ESCEO 2019): ESCEO-IOF USA Symposium Abstracts
}

\author{
ESCEO-IOFUSA1 \\ RECOMMENDATIONS FOR THE CONDUCT OF \\ ECONOMIC EVALUATIONS IN OSTEOPOROSIS: A \\ SUMMARY FROM AN ESCEO-IOF USA WORKING \\ GROUP \\ M. Hiligsmann ${ }^{1}$ \\ ${ }^{1}$ Department of Health Services Research, CAPHRI Care and \\ Public Health Research Institute, Maastricht University, \\ Maastricht, The Netherlands
}

Objective: To provide recommendations for the conduct of economic evaluations in osteoporosis in order to improve their transparency, comparability, and methodologic standards.

Material and Methods: An ESCEO-IOF USA working group (including 23 experts) was convened to make recommendations for the design, conduct, and reporting of economic evaluations in osteoporosis, and to define an osteoporosisspecific reference case to serve a minimum standard for all economic analyses in osteoporosis. A literature review, a faceto-face meeting in New York City (including 11 experts), and a review/approval by a larger group of experts worldwide (including 23 experts in total) were conducted.

Results: Recommendations on the type of economic evaluation, methods for economic evaluation, modeling aspects, base-case analysis and population, excess mortality, fracture costs and disutility, treatment characteristics, and model validation were provided. Recommendations for reporting economic evaluations in osteoporosis were also made and an osteoporosis-specific checklist was designed that includes items to report when performing an economic evaluation in osteoporosis. Further, 12 minimum criteria for economic evaluations in osteoporosis were identified.

Conclusion: These recommendations are intended to supplement general and national guidelines for economic evaluations, improve transparency, quality, and comparability of economic evaluations in osteoporosis, and maintain methodologic standards to increase their use by decisionmakers.

\section{ESCEO-IOFUSA2}

CHALLENGES, UNCERTAINTIES AND FUTURE RESEARCH AGENDA FOR THE CONDUCT OF ECONOMIC EVALUATIONS IN OSTEOPOROSIS: A SUMMARY FROM AN ESCEO-IOF USA WORKING GROUP

S. Silverman $^{1}$

${ }^{1}$ Cedars-Sinai Medical Center, UCLA School of Medicine and the, OMC Clinical Research Center, Los Angeles, United States

Objective: To identify methodologic challenges, uncertainty and initiate a call for future research for the conduct of economic evaluation in osteoporosis.

Material and Methods: An ESCEO-IOF USA working group (including 23 experts) was convened to discuss methodologic challenges and initiate a call for research on economic evaluation in osteoporosis.

Results: Several methodologic challenges were identified and a call for future research was suggested in the following areas: 1 . Modeling fracture risk after 10 years; 2 . Understanding the increased risk of fracture after a fracture; 3 . Excess mortality after non-hip non-vert fracture; 4 . Long term costs of care after hip fracture; 5 . Understanding societal vs payor perspectives; 6 . Modeling multiple fractures and their severity within the model; 8. Identifying appropriate comparators; 9. Absence of hip fracture data with some therapies; 10 . Persistence of treatment effect after discontinuation; 11. Variations in response based on prior treatment exposure: 12 . Treatment side effects; 12 . Lack of sufficient data in males; 13. Cost effectiveness in subpopulations. Conclusion: While the working group acknowledges challenges and the need for further research on economic evaluation in osteoporosis, this research may help increase the use of cost-effectiveness analyses by decision makers and to lead to a more effective allocation of health resources.

Publisher's Note Springer Nature remains neutral with regard to jurisdictional claims in published maps and institutional affiliations. 